\title{
Multifractal characterization of epitaxial silicon carbide on silicon
}

\author{
Ţălu ŞTefan ${ }^{1}$, Stach Sebastian $^{2}$, RAmaZanov Shikhgasan $^{3,4}$, Sobola Dinara $^{5, *}$, \\ RAMAZANOV GUSEYN ${ }^{4}$ \\ ${ }^{1}$ Technical University of Cluj-Napoca, Faculty of Mechanical Engineering, Department of AET, Discipline of Descriptive \\ Geometry and Engineering Graphics, 103-105 B-dul Muncii St., Cluj-Napoca 400641, Cluj, Romania \\ ${ }^{2}$ University of Silesia, Faculty of Computer Science and Materials Science, Institute of Informatics, Department of \\ Biomedical Computer Systems, ul. Będzińska 39, 41-205 Sosnowiec, Poland \\ ${ }^{3}$ SICLAB Limited liability company, 367030 Makhachkala, M. Yaragskogo 75, Dagestan Republic, Russia \\ ${ }^{4}$ Dagestan State University, Faculty of Physics, 367015 Makhachkala, M. Gadjieva 43-a, Dagestan Republic, Russia \\ ${ }^{5}$ Brno University of Technology, Faculty of Electrical Engineering and Communication, Physics Department, Technická 8 , \\ 61600 Brno, Czech Republic
}

\begin{abstract}
The purpose of this study was to investigate the topography of silicon carbide films at two steps of growth. The topography was measured by atomic force microscopy. The data were processed for extraction of information about surface condition and changes in topography during the films growth. Multifractal geometry was used to characterize three-dimensional micro- and nano-size features of the surface. X-ray measurements and Raman spectroscopy were performed for analysis of the films composition. Two steps of morphology evolution during the growth were analyzed by multifractal analysis. The results contribute to the fabrication of silicon carbide large area substrates for micro- and nanoelectronic applications.
\end{abstract}

Keywords: surface roughness; multifractal analysis; atomic force microscopy; silicon carbide; film growth

\section{Introduction}

\section{1. $\mathrm{SiC} / \mathrm{Si}$ hybrid substrates}

Incorporation of silicon carbide (SiC) into silicon $(\mathrm{Si})$ technologies is a chance for improvement of electronic performance. $\mathrm{SiC}$ is considered as a good anti-oxidation coating [1]. It is a key material for III-V compound LED substrates where surface quality is one of the factors defining efficiency [2]. Si-terminated SiC substrate was described in [3] as a material for fabrication of clean graphene lattice. $\mathrm{Si} / \mathrm{SiC}$ heterojunction could find application in $\mathrm{SiC}$ power devices activated by nonultraviolet light [4]. Radiation, thermal, electrical, mechanical and chemical stability of SiC gives advantages to $\mathrm{Si} / \mathrm{SiC}$ hybrid substrates. An inverse task of $\mathrm{Si} / \mathrm{SiC}$ heterostructures preparation was described by Lianbi et al. [4], Long-Fei et al. [5] and

*E-mail: sobola@vutbr.cz growth of silicon on silicon carbide substrates was demonstrated. The differences in thermal expansion coefficients and crystal lattice mismatch make fabrication of these structures troublesome and impair performance of the devices [6].

\subsection{Fractal approach to surface charac- terization}

Fractal and kinetic approach has been of interest for description of surface growth, agglomeration and other type of structure evolution, such as cluster formation [7], for more than 20 years. The fractal dimension was studied as a function of morphology. Morphological properties of surface features are related to film thickness. Many studies have been dedicated to $\mathrm{SiC} / \mathrm{Si}$ structures, but according to our review, multifractal characterization of $\mathrm{SiC}$ on $\mathrm{Si}$ has not been actually presented yet. In this paper, we describe multifractal approach to the growth of thin films by magnetron sputtering 
on the example of SiC films. Roughness and texture play a key role in material description and the role of the multifractal values is specification of topography appearance. The topography characterization techniques extract significant surface parameters and are consolidated with the models and theories of material behavior [8].

The fractal geometry allows extraction of highly distinctive features from 3-D geometric patterns of surfaces, as compared with traditional statistical parameters [9-15]. The fractal analyses allow an easy realization, certainty and fast time of calculation [15-18]. The multifractal geometry offers a new way to evaluate the local and global degree of 3-D surface irregularities in quantitative and qualitative ways [10-14].

Commonly, the fractal dimension $\mathrm{D}$ of a surface is a value ranging within $2 \leqslant D \leqslant 3$, where $\mathrm{D}=2$ for smooth surface and $D=3$ for very rough surface. A higher value of this variable corresponds to a higher level of irregularity and roughness.

Ramazanov et al. [19] have mentioned in a recent study about the growth surface evolution during epitaxy of silicon carbide on silicon using the fractal geometry with morphological envelopes method. The aim of this study was to acquire qualitative and quantitative nanoscale data of the 3-D surface micromorphology of $\mathrm{SiC}$ epilayers through multifractal analysis.

\subsection{Relation to previous studies}

This work is a continuation of two papers [19, 20]. The first article describes technological process of $\mathrm{SiC}$ preparation on $\mathrm{Si}$ substrates by magnetron sputtering. Greater epilayers perfection was achieved with increasing of film thickness. The study of morphology was carried out at different steps of film growth by atomic force microscopy. The results were used for a study of fractal dependence of surface evolution on the growth time. The other article describes the dependences between fractals (mathematical analysis of films surface morphology) and mechanism of growth for highly mismatched substrates.
In present article, we examine the surface features in more detail. The quality of the films is studied by X-ray and Raman measurements.

\section{Experimental}

\subsection{Materials}

Silicon carbide polycrystalline target was sputtered on properly prepared commercially available silicon substrates (with orientation of surface deflected from (lll $\left.1 \begin{array}{ll}1 & 1\end{array}\right)$ plane). Temperature of the films preparation was $950{ }^{\circ} \mathrm{C}$ to $1100{ }^{\circ} \mathrm{C}$. The chamber was evacuated to a vacuum of $1.33 \times 10^{-4} \mathrm{~Pa}$ before starting the flow of inert gas (Ar) for sputtering the target material. The samples were taken from the chamber after cooling to room temperature. The technology details were reported in $[19,20]$. Since silicon carbide does not oxidize in air, the exposition of the samples to the air did not influence its characteristics. The thicknesses of the films were measured by observing the samples crossections using scanning electron microscope. $\mathrm{X}$-ray diffraction data proved the presence of 3C-SiC cubic modification (Fig. 1).
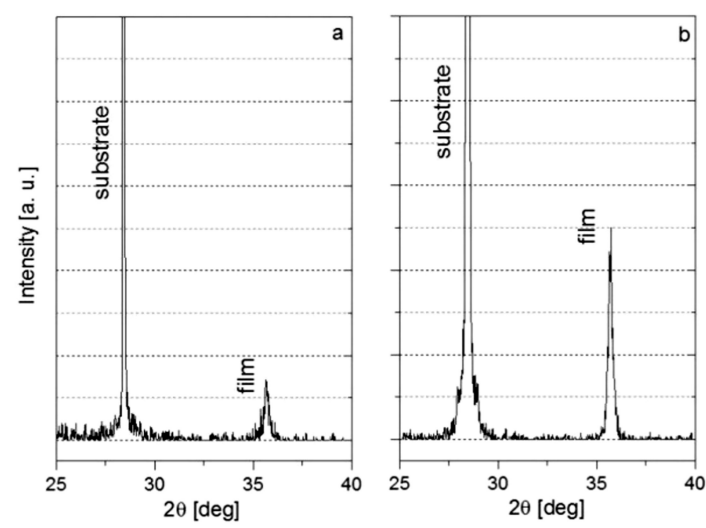

Fig. 1. Diffraction pattern of 3C-SiC/Si(l 111 ) structure: for film thickness (a) $140 \mathrm{~nm}$ and (b) $420 \mathrm{~nm}$.

Raman spectra of the samples were measured for more detailed study of the influence of changing process parameters on crystal lattice of the film and substrate at their interface (Fig. 2).

Raman spectra were investigated by Ntegra Spectra instrument with a solid-state laser 


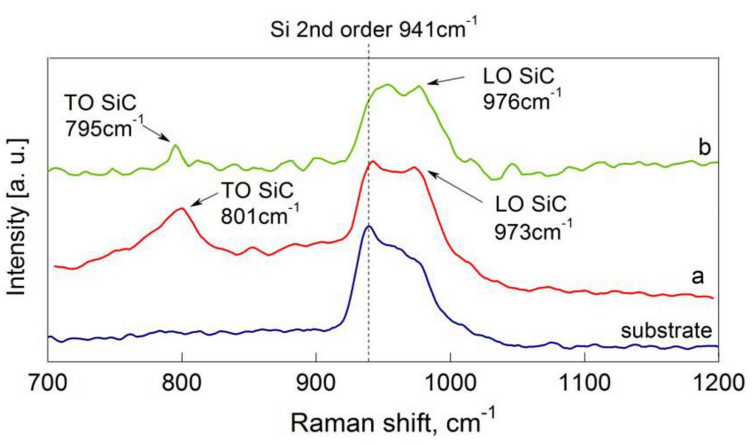

Fig. 2. Raman displacement of SiC/Si structure: (a) at the first stage of $\mathrm{SiC}$ growth at $120 \mathrm{~nm}$ thickness, (b) growth of $\mathrm{SiC}$ film at $420 \mathrm{~nm}$ thickness.

(wavelength $\lambda=633 \mathrm{~nm}$ ). Surface topography of a film can be characterized by a number of methods. We chose AFM since it gives real 3D topography data of surface features.

The displacement of the Raman spectra indicates that the peak of optical mode TO (at the area $801 \mathrm{~cm}^{-1}$ ) moves by $\sim 6 \mathrm{~cm}^{-1}$ after increasing the film thickness by three times. At the same time the acoustic mode LO (at the area of $973 \mathrm{~cm}^{-1}$ ) moves by $\sim 3 \mathrm{~cm}^{-1}$. It is caused by transformation of crystal lattice into a standard cubic modification $\beta$-SiC.

AFM provides quantitative and qualitative data about surface morphology. Semi-contact AFM is widely used for precise description of shapes and sizes of surface features. The analysis of fractal geometry requires well measured topography data for characterization of morphological structures [21].

AFM has better resolution than traditional instruments and it can be adapted for measurements of bare and untreated surfaces without complicated sample preparations. Precise information about the height of surface features could be extracted (Fig. 3). The sample is not damaged by high energy beam as could happen in scanning electron or ion methods.

\subsection{Multifractal analysis of the grown layers}

The multifractal analysis as a generalization of fractal approach can be applied to study the local affinity of topography features. It is useful when

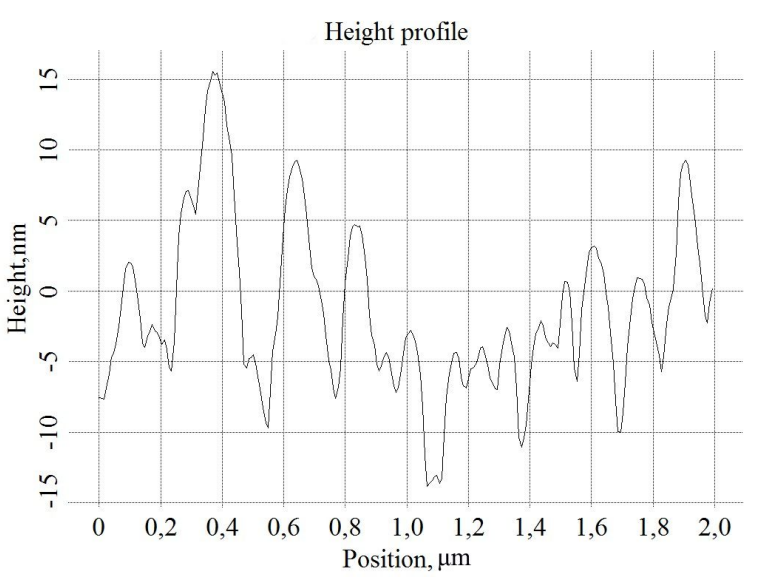

(a)

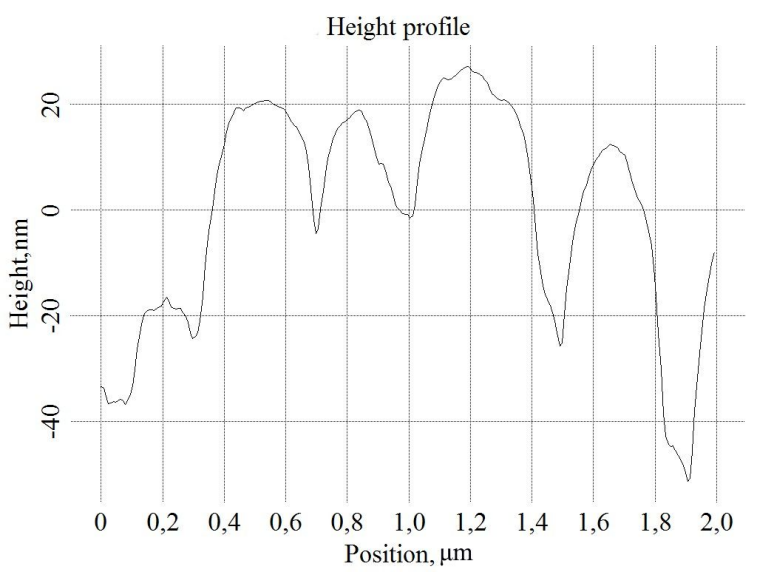

(b)

Fig. 3. Profile of AFM measurements: (a) film thickness $140 \mathrm{~nm}$ and (b) film thickness $420 \mathrm{~nm}$.

a single fractal measure does not describe the topography elements of films and coatings.

Two common multifractal measures are used in computational analyses: the generalized dimension Dq of the qth order moment of a distribution and the $f(\alpha)$ singularity spectrum. The generalized dimension Dq could be determined for any real parameter $\mathrm{q}$ in the range from $-\infty$ (concentrates on less dense regions) to $+\infty$ (concentrates on dense regions) that shows the order of the measure moment. $\alpha(q)$ is called Hölder exponent of the qth order moment or singularity exponent.

Furthermore, these two multifractal measures can be connected with the Legendre transformation. The generalized dimensions, $\mathrm{Dq}$ for $\mathrm{q}=0$, 
$\mathrm{q}=1$ and $\mathrm{q}=2$, are known as the capacity (or box-counting), the information (or Shannon) entropy and correlation dimensions, respectively.

For a multifractal surface, all dimensions are different and the relationship between $\mathrm{D}_{0}, \mathrm{D}_{1}$, and $\mathrm{D}_{2}$ is, $\mathrm{D}_{0}>\mathrm{D}_{1}>\mathrm{D}_{2}$. For $\mathrm{q}= \pm \infty, \alpha_{\min }=\mathrm{D}_{+\infty}$ and $\alpha_{\max }=\mathrm{D}_{-\infty}$ (where $\mathrm{D}_{-\infty}$ and $\mathrm{D}_{+\infty}$ are the limits of the generalized dimension spectrum).

The multifractal width spectrum is defined as $\Delta \alpha=\alpha_{\max }-\alpha_{\min }$, and the spectrum arms' heights difference is computed with the formula: $\Delta \mathrm{f}=\mathrm{f}\left(\alpha_{\min }\right)-\mathrm{f}\left(\alpha_{\max }\right)$.

The left arm of the multifractal spectrum, defined by positive $\mathrm{q}$ values, is associated with strongly irregular areas; whereas the right arm of the multifractal spectrum, defined by negative $q$ values, is associated with flat areas.

A 3-D complex multifractal structure is considered if the Dq value decreases gradually with an increase of q. The multifractal analyses depend on the multifractal analysis methods, including the algorithm and specific calculation used.

In our study, by applying the multifractal analysis based on the box counting method in each region of interest (ROI), the generalized dimension and singularity spectrum are defined by the functional dependences Dq versus $q$ and $f(\alpha)$ versus $\alpha$.

The AFM data from the measured samples were processed using the watershed transform based on the Mountains Map ${ }^{\circledR} 7$ Software (Digital Surf, Besançon, France).

\subsection{Statistical analysis}

Statistical analysis of the AFM data was done by SPSS 14 for Windows (Chicago, Illinois, USA). One-way analysis of variance was used to test the differences of the samples with two different widths with Scheffé post hoc tests for multiple comparisons. The level of significance was set at $\mathrm{P}<0.05$.

\section{Results}

In the studies, two samples with different thicknesses: F1 $140 \mathrm{~nm}$ and R1 $420 \mathrm{~nm}$ were used. The samples were scanned by AFM four times, for different square areas: $2 \mu \mathrm{m} \times 2 \mu \mathrm{m}, 5 \mu \mathrm{m} \times 5 \mu \mathrm{m}$, $10 \mu \mathrm{m} \times 10 \mu \mathrm{m}$, and $20 \mu \mathrm{m} \times 20 \mu \mathrm{m}$.

The representative 3-D AFM images of scanned square areas $(2 \mu \mathrm{m} \times 2 \mu \mathrm{m}, 5 \mu \mathrm{m} \times 5 \mu \mathrm{m}$, $10 \mu \mathrm{m} \times 10 \mu \mathrm{m}, 20 \mu \mathrm{m} \times 20 \mu \mathrm{m})$ of the $\mathrm{SiC}$ epilayer surface nanostructures for samples (F1 and R1) show an inhomogeneous structure (Fig. 4 and Fig. 5).

The multifractal singularity spectra $f(\alpha)$ calculated for all nanostructures presented in Fig. 4 and Fig. 5 are graphically illustrated in Fig. 6 and Fig. 7, in which the $f(\alpha)$ spectra were computed in the range $-10 \leqslant q \leqslant 10$ for successive 1.0 steps.

In Fig. 6 and Fig. 7, the curve f(q,r) to $\alpha(\mathrm{q}, \mathrm{r})$ associated with each group is a characteristic curve with a specific shape (non-linear and asymmetric), and with different width spectrum $\Delta \alpha$, (invariably with two arms).

The left and right shoulder of the multifractal singularity spectrum $f(\alpha)$ for the 3-D surface of group \#F1 $2 \mu \mathrm{m} \times 2 \mu \mathrm{m}$ is much longer than those of all the other samples (Fig. 6). On the other hand, the right shoulder of the multifractal singularity spectrum $f(\alpha)$ for the surface of the group \#F1 $10 \mu \mathrm{m} \times 10 \mu \mathrm{m}$ is much shorter than those of all the other samples, respectively (Fig. 6).

The values of generalized fractal dimensions $\mathrm{Dq}$ (for $\mathrm{q}=0,1,2$ ), the multifractal width spectrum $\Delta \alpha=\alpha_{\max }-\alpha_{\min }$, and the spectrum arms heights difference $\Delta \mathrm{f}=\mathrm{f}\left(\alpha_{\min }\right)-\mathrm{f}\left(\alpha_{\max }\right)$ were calculated.

AFM images of all scanned areas of the samples reveal for every group a specific three-dimensional topography. They also can be described by multifractal measures. The generalized dimensions, $\mathrm{D}_{0}>\mathrm{D}_{1}>\mathrm{D}_{2}$, differ for all groups. It confirms the multifractal nature of topography of the sample surfaces. There is a specific distribution of surface characteristics (in amplitude, spatial distribution and pattern) of local and global character. A summary of the statistical parameters for F1 


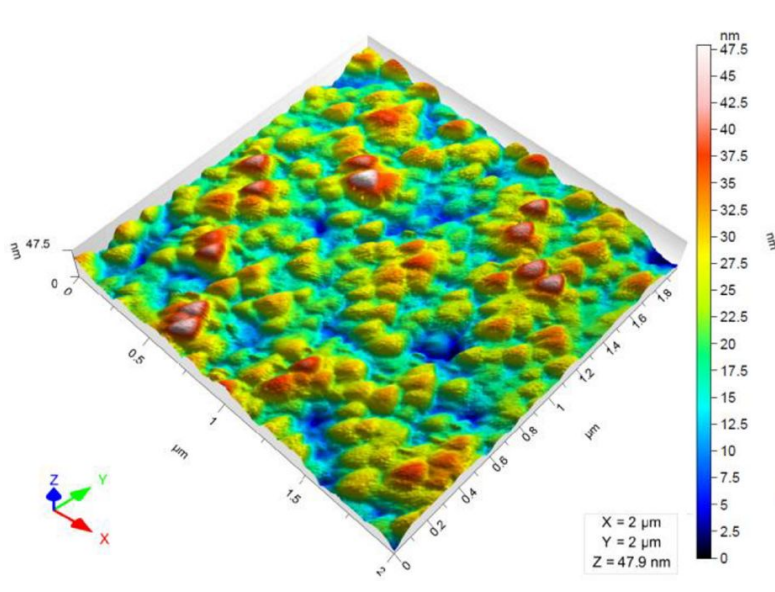

(a)

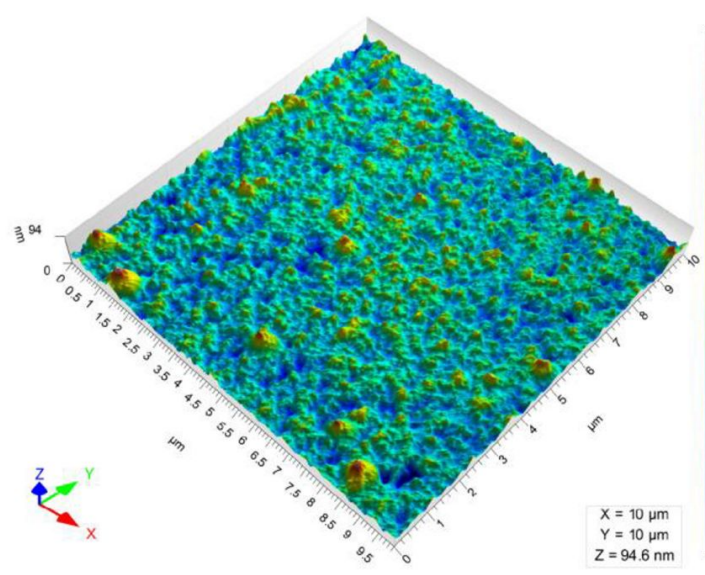

(c)

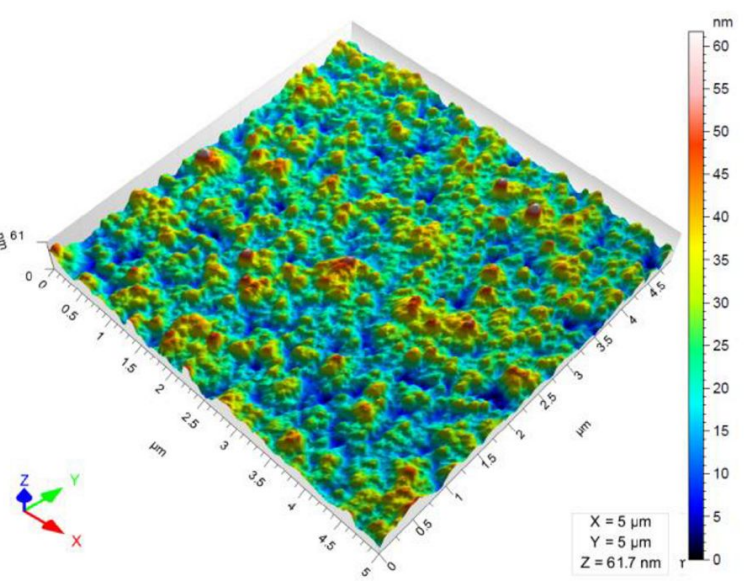

(b)

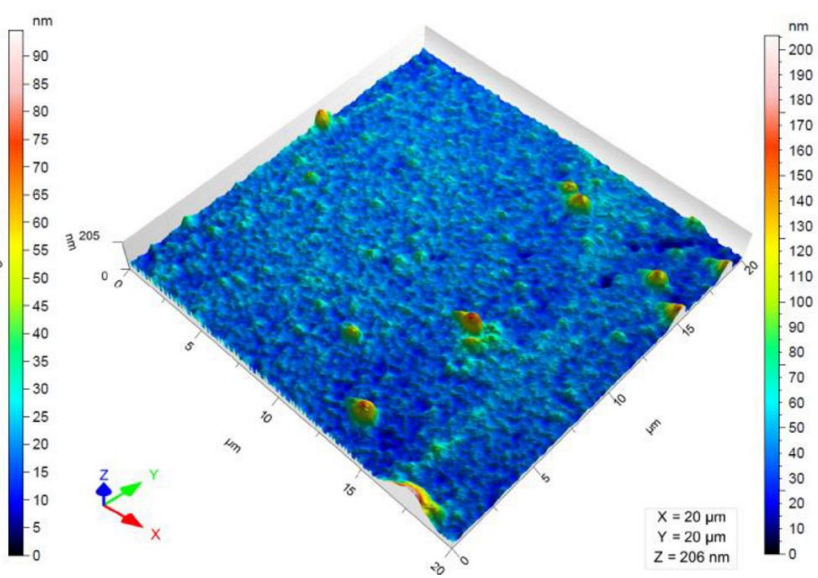

(d)

Fig. 4. A set of representative 3-D AFM images of F1 nanostructure surfaces: (a) F1 $2 \mu \mathrm{m} \times 2 \mu \mathrm{m}$, (b) F1 $5 \mu \mathrm{m} \times 5 \mu \mathrm{m}$, (c) F1 $10 \mu \mathrm{m} \times 10 \mu \mathrm{m}$, and (d) F1 $20 \mu \mathrm{m} \times 20 \mu \mathrm{m}$.

$20 \mu \mathrm{m} \times 20 \mu \mathrm{m}$ and $\mathrm{R} 120 \mu \mathrm{m} \times 20 \mu \mathrm{m}$ results was done according to ISO 25178-2 [22].

\section{Discussion}

$\mathrm{SiC}$ films heterostructures with two thicknesses were prepared on silicon substrate by magnetron technique. The 3-D topography of analyzed samples at low size scale has been resolved by probe microscopy data and their multifractal analysis.

Strong covalent bond atoms of silicon carbide form angular grains which are observed in small scanning areas (Fig. 4a and Fig. 5a) and, as it is shown in the AFM images, the small grains are merged into larger features of the same shape for the larger film thickness.
The film surface of group \#F1- $2 \mu \mathrm{m} \times 2 \mu \mathrm{m}$ has the most irregular topography $(\Delta \alpha=0.6894$, value larger than $\Delta \alpha$ values of all the other samples). On the other hand, the most regular film surface is found for the film surface of group \#F1 $10 \mu \mathrm{m} \times 10 \mu \mathrm{m}(\Delta \alpha=0.3855)$. The same behavior is observed for \#R1 $2 \mu \mathrm{m} \times 2 \mu \mathrm{m}(\Delta \alpha=1.3002$ value bigger than $\Delta \alpha$ values of all the other samples). It is caused by the fact that the small scan areas have been chosen on the large scan squares at relatively smooth and homogeneous places.

Opposite to \#F1, the most regular surface is observed for the largest scanning area for \#R1 $20 \mu \mathrm{m} \times 20 \mu \mathrm{m}(\Delta \alpha=0.8548)$. The topography of \#F1 sample still reveals the substrate features 


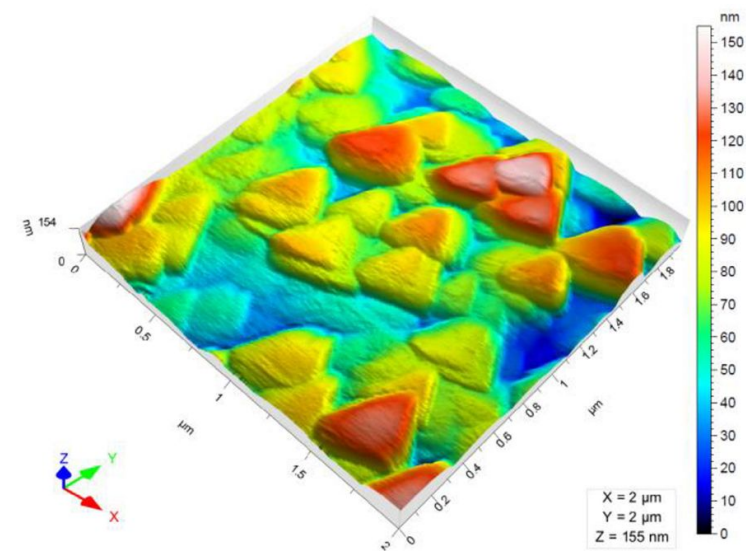

(a)

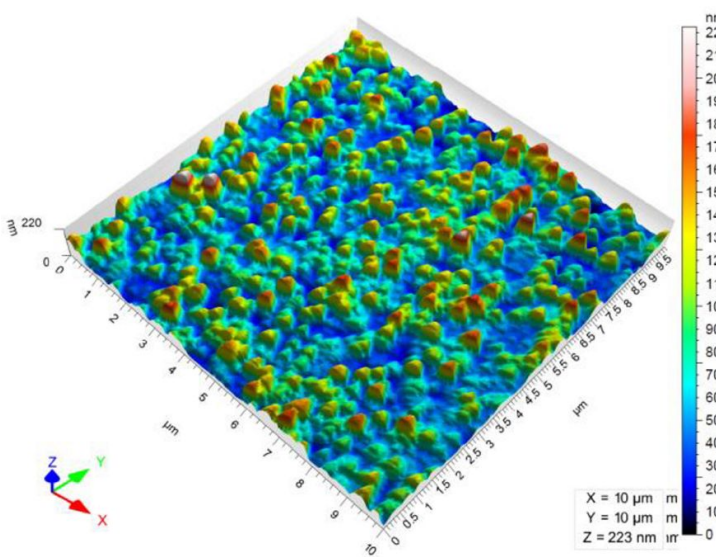

(c)

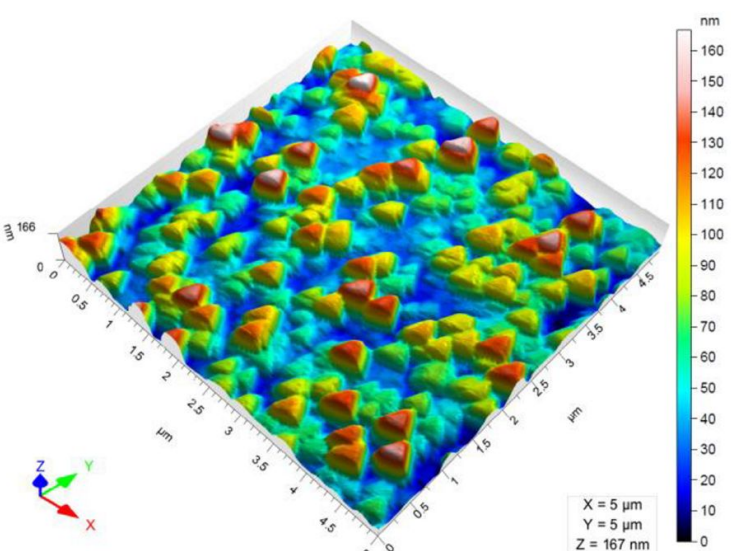

(b)

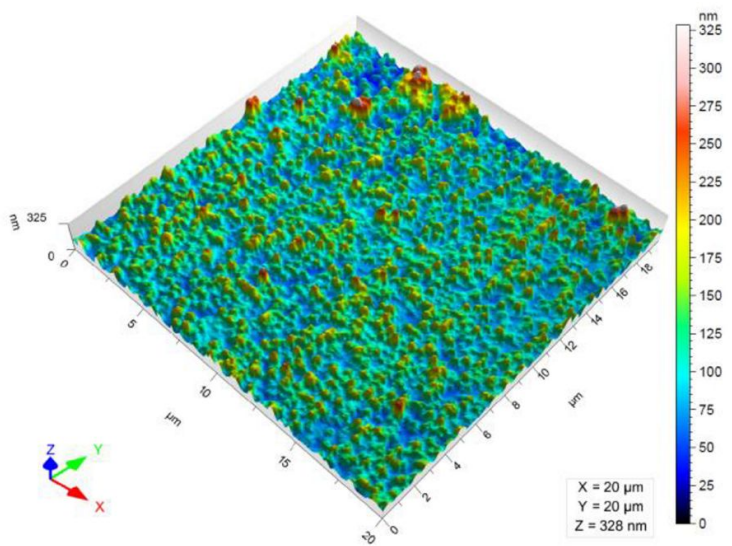

(d)

Fig. 5. A set of representative 3-D AFM images of R1 the nanostructure surfaces: (a) R1 $2 \mu \mathrm{m} \times 2 \mu \mathrm{m}$, (b) R1 $5 \mu \mathrm{m} \times 5 \mu \mathrm{m}$, (c) R1 $10 \mu \mathrm{m} \times 10 \mu \mathrm{m}$, and (d) R1 $20 \mu \mathrm{m} \times 20 \mu \mathrm{m}$.

and represents earlier steps of film growth. So, \#R1 sample with thicker $\mathrm{SiC}$ film is supposed to be more regular at the larger scan areas.

Quantitative analysis of statistical parameters reveals that the sample \# F1 $20 \mu \mathrm{m} \times 20 \mu \mathrm{m}$ has much larger height, hybrid, and functional parameters (volume) than the sample \#R1 $20 \mu \mathrm{m} \times 20 \mu \mathrm{m}$. Furthermore, the left arm of the multifractal spectrum corresponds to strongly irregular areas, defined by a high dimension value (Fig. 6 and Fig. 7).

As can be seen for all groups, $\Delta \alpha>0$ and $\Delta \mathrm{f}<0$. This can be explained by overgrowth of silicon substrate imperfections: the growing film of silicon carbide spreads along the surface by islands coalescence which have the same shape. A scheme of these growth phenomena is shown in Fig. 8.

Surface and grain boundary diffusion is determined by size distribution of the grains and the shape could be explained by tetrahedral form of the elementary $\mathrm{SiC}$ lattice. The coalescence of grains is responsible for void-free surface. A fractal dimension of the \#R1 sample is lower in common because the grains on the surface are larger.

The small area scans demonstrate "zoomed" grains: the isotropy of \#F1 small scans is twice higher than for \#R1 small scans. In this case isotropy (similarity) could be explained by coalescence of random islands that look quite similar. So, there must be similar small topography features 


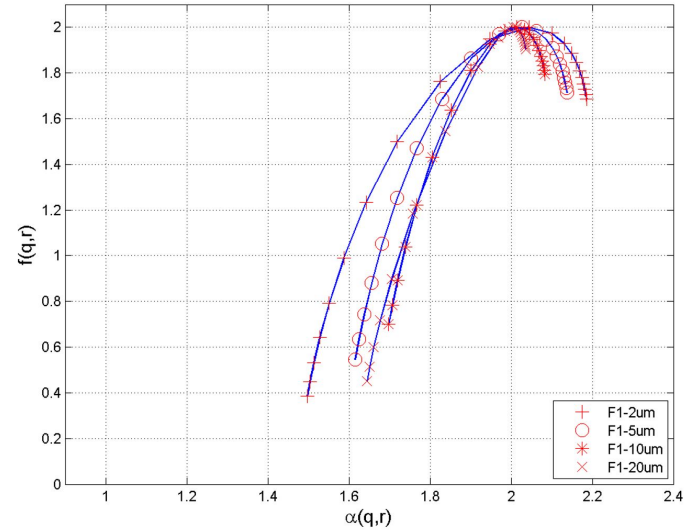

Fig. 6. Multifractal singularity spectra $f(\alpha)$ for $F 1$ nanostructure surfaces: (a) F1 $2 \mu \mathrm{m} \times 2 \mu \mathrm{m}$, (b) F1 $5 \mu \mathrm{m} \times 5 \mu \mathrm{m}$, (c) F1 $10 \mu \mathrm{m} \times 10 \mu \mathrm{m}$, and (d) F1 $20 \mu \mathrm{m} \times 20 \mu \mathrm{m}$.

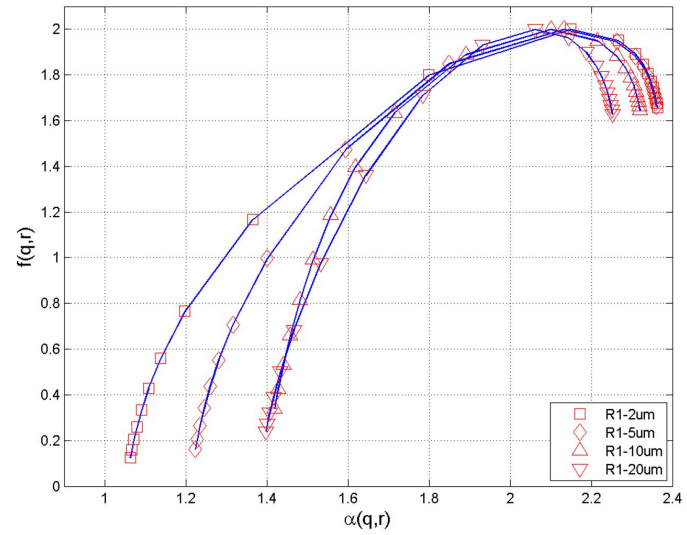

Fig. 7. Multifractal singularity spectra $f(\alpha)$ for R 1 nanostructure surfaces: (a) R1 $2 \mu \mathrm{m} \times 2 \mu \mathrm{m}$, (b) $\mathrm{R} 15 \mu \mathrm{m} \times 5 \mu \mathrm{m}$, (c) R1 $10 \mu \mathrm{m} \times 10 \mu \mathrm{m}$, and (d) R1 $20 \mu \mathrm{m} \times 20 \mu \mathrm{m}$.

which merge into larger morphology elements with further growth of the film thickness.

However, isotropy of \#F1 surface is lower for large scan images and isotropy of \#R1 is higher for large scan images. The large scan areas of the samples (where the grains are not well recognizable and the topography on the whole is estimated) indicate that isotropy of thicker film is higher because the film becomes more homogeneous during the growth on large squares of the substrate surface. 3C-SiC films become highly-oriented during

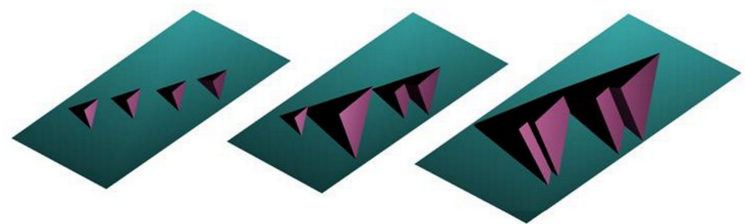

Fig. 8. Simple scheme of 3C-SiC growth.

the growth evolution because of strain energy relaxation with film thickness growth.

The results of films growth morphology demonstrate adaptability of multifractal approach, but further studies on a large amount of samples are necessary for establishment of precise dependences. The roughness and thickness of the films can be adjusted by controlling the sputtering time.

Ionized atoms of silicon and carbon in the process of magnetron sputtering arrive at the surface of the growing film with larger part of their initial energy (lower than $\mathrm{Ei}=20 \mathrm{eV}$ ). As a result, they provide a significant part of energy to the growth of the $\mathrm{SiC}$ film. It makes the kinetic processes faster on the substrate surface. Ionized carbon atoms tie together the crystal lattice parallel to the growing layer because these atoms have high binding energy with atoms of $\operatorname{Si}\left(\begin{array}{lll}1 & 1 & 1\end{array}\right)$ substrate and create $\mathrm{C}-\mathrm{C}$ bonds. Dislocations create an interface between the film and substrate for removing the elastic deformations. Shift of nodes occurs in crystal lattice in the direction of $3 \mathrm{C}-\mathrm{SiC}$ film growth. It reduces excessive mechanical stresses at the heterojunction.

Raman spectra show that the wide peak in the range of $942 \mathrm{~cm}^{1}$ to $973 \mathrm{~cm}^{-1}$ in LO mode (belonging to Si substrate) shifts in the junction area of $2 \mathrm{~cm}^{-1}$ in relation to the volume peak (Fig. 2, spectrum a), and the $973 \mathrm{~cm}^{-1}$ peak observed in the area of $\mathrm{SiC}$ film begins to grow. The peak of TO mode shifts by $3 \mathrm{~cm}^{-1}$ with maximum of TO phonon at $796 \mathrm{~cm}^{-1}$ for 3C-SiC film in \#R1 sample (Fig. 2, spectrum b).

The wide peak which corresponds to LO mode $\mathrm{Si}+3 \mathrm{C}-\mathrm{SiC}$ in \#R1 sample displaces from $954 \mathrm{~cm}^{1}$ to $976 \mathrm{~cm}^{-1}$ (Fig. 2 spectrum b) and with maximum of LO mode 3C-SiC $976 \mathrm{~cm}^{-1}$ [23]. This peak belongs to high quality film of $3 \mathrm{C}-\mathrm{SiC}$. 
Intensity of $\mathrm{TO}$ mode of $3 \mathrm{C}-\mathrm{SiC}$ for \#R1 sample falls because of increasing the film thickness, but the value of half-width of the peak is improved in relation to the film of \#F1 sample. An intense peak is observed in the short-wave Raman spectrum of the substrate at $516 \mathrm{~cm}^{-1}$ in sample \#F1 and it is shifted to $522 \mathrm{~cm}^{-1}$ in sample \#R1. This effect can be explained by monoaxial stretching of the silicon lattice in direction $\left\langle\begin{array}{lll}1 & 1 & 1\end{array}\right\rangle$ due to the rising energy of 3C-SiC film with increasing film thickness. Displacement of $\mathrm{TO}$ mode for $3 \mathrm{C}-\mathrm{SiC}$ films of different thicknesses and improving of quality $\Delta \mathrm{FWHM}=19 \mathrm{~cm}^{-1}$ indicates the relaxation of macro-stresses along the thickness on the growing film.

\section{Conclusions}

Multifractal spectrum study is a tool to evaluate a process of film deposition from mathematical point of view since the multifractal spectrum provides a link to information on the physical processes which take place during film deposition, such as coalescence of grains - the reason of features occurrence (influence on the substrate morphology, etc.).

The results of processing AFM data of $\mathrm{SiC}$ on $\mathrm{Si}$ substrates have been presented for the samples with different thicknesses. Atomic force microscopy and multifractal analysis describe uniformity of morphology on micro- and nanoscale. Multifractal analysis gives qualitative and quantitative information about morphology evolution during the film growth. It is necessary to take into account the size of AFM scans, since different scan sizes reveal either very precise data about the elemental composition or the appearance of the topography as a whole. The data processed by multifractal analysis are connected with the fabrication parameters (time of deposition) and composition of obtained samples. The presented results are correlated to previous studies where we noted that 3C-SiC films became highly oriented during growth evolution because of strain energy relaxation with the growth of film thickness.
The results contribute to the films preparation. The multifractal characteristics are connected with the stage of films growth. The multifractal metrics could be applied for description of other films morphology with similar mechanism of growth. The application of multifractal characterization is useful in mechanical stress and defect studies of epitaxial films.

\section{Acknowledgements}

Research described in the paper was financially supported by the Ministry of Education, Youth and Sports of the Czech Republic under the project CEITEC 2020 (LQ1601), by the Grant Agency of the Czech Republic under No. GACR 1505259S and by the National Sustainability Program under the Grant LO1401. For the research, infrastructure of the SIX Center was used.

\section{References}

[1] Li B., Kang P., Gou H,, Wu G., Mula S., Corros. Sci., 88 (2014), 473.

[2] Zhou Y., Pan G., Shi X., Xu L., Zou CH., Gong H., Luo G., Appl. Sur. Sci., 316 (2014), 643.

[3] LiU X., Han Y., Evans J.W., Engstfeld A.K., Juergen Behm R., Tringides M.C., Hupalo M., Lin H., Huang L., Ho K., Appy D., Thiel P.A., WANG C., Prog. Sur. Sci., 90 (2015), 397.

[4] Lianbi L., Zhiming CH., Longfei X., Chen Y., Mater. Lett., 93 (2013), 330.

[5] Long-Fei X., Zhi-Minga CH., Lian-Biab L., Chena Y., Xiao-Min H., Na Y., Appl. Sur. Sci., 261 (2012), 88

[6] Anzalone R., Alberti A., Lavia F., Mater. Lett., 118 (2014), 130.

[7] Chaiken J., Goodisma J., Photochem. Photobiol. A, 80 (1994), 53.

[8] Vasilev B., Bott S., RzehaK R., Liske R., BARTHA J.W., 104 (2013), 48.

[9] Dallaeva D., Ţălu Ş., Stach S., ŠKarvada P., Tománek P., Grmela L., Appl. Sur. Sci., 312 (2014), 81.

[10] Ţălu Ş., Stach S., Mahajan A., Pathak D., WagNER T., Kumar A., Bedi R.K., ŢăLU M., Electron. Mater. Lett., 10 (2014), 719.

[11] Ţ̌Llu Ş., Ghazai A.J., STACH S., Hassan A., HaSSAN Z., ŢĂLU M., J. Mater. Sci. Mater. El., 25 (2014) 466.

[12] Ţălu Ş., Stach S., Valedbagi S., Elahi S.M., BAVADI R., Mater. Sci.-Poland, 33 (2015), 137.

[13] Ţălu Ş., BRamowicz M., Kulesza S., Solaymani S., Ghaderi A., Dejam L., Elahi S.M., Boochani A., Superlattice. Microst., 93 (2016), 109.

[14] ŢĂLU Ş., Micro and nanoscale characterization of three dimensional surfaces. Basics and applications, Napoca Star Publishing House, Cluj-Napoca, Romania, 2015. 
[15] ŢăLU Ş., SOlaYmani S., BRAMOWICZ M., Naseri N., Kulesza S., Ghaderi A., RSC $A d v ., 6$ (2016), 27228.

[16] ŢĂLU Ş., BRAmowicz M., Kulesza S., Ghaderi A., Dalouji V., Solaymani S., Fathi Kenari M., Ghoranneviss M., J. Microsc., 264 (2016), 143.

[17] ŢĂLU Ş., BRAmowicz M., Kulesza S., Dalouji V., Solaymani S., Valedbagi S., Microsc. Res. Tech., 79 (2016), 1208.

[18] ŢĂLU Ş., BRAmowicz M., Kulesza S., GHaderi A., Solaymani S., SAVAloni H., BABAei R., J. Ind. Eng. Chem., 43 (2016), 164.

[19] Ramazanov S., Ţălu Ş., Sobola D., Stach, S., Ramazanov G., Superlattice. Microstruct., 86 (2015), 395.
[20] Ramazanov SH. M., Ramazanov G. M., Tech. Phys. Lett., 40 (2014), 44.

[21] Wucherpfennig TH., Lakowitz A., Krull R., J. Biotechnol., 163 (2013), 124.

[22] ISO 25178-2: 2012, Geometrical product specifications (GPS) Surface texture: Areal Part 2: Terms, definitions and surface texture parameters. Available from: http: //www . iso.org (accessed on March 10, 2017).

[23] Kladko V.P., Kuchuk A.V., SafryUK N.V., Machulin V.F., Belyaev A.E., Hardtdegen H., Vitusevich S.A., Appl. Phys. Lett., 95 (2009), 031907.
Received 2016-12-13 Accepted 2017-03-28 